\title{
1 Lungenfunktion und pulmonaler Gasaustausch
}

\author{
Peter Neumann
}

Adipositas ist mit vielfältigen Veränderungen der Lungenfunktion assoziiert. Diese Veränderungen betreffen Atemmechanik, Gasaustausch sowie Atemantrieb und werden zum Teil direkt auf das Übergewicht, zum Teil aber auch auf die verschiedenen Begleiterkrankungen der Adipositas zurückgeführt. Adipöse Patienten haben perioperativ ein erhöhtes Risiko für Atemwegs- und pulmonale Komplikationen, sodass profunde Kenntnisse der pathophysiologischen Veränderungen des respiratorischen Systems bei Adipositas für Intensivmediziner und Anästhesisten unabdingbar sind. Übergewicht führt zu einer gewichtsabhängigen Reduktion der funktionellen Residualkapazität (FRC) und des exspiratorischen Reservevolumens (ERV). Dadurch kann besonders in den basalen Lungenabschnitten schon während einer normalen Ausatmung ein Verschluss von Bronchiolen auftreten, was zu einer Abnahme der Ventilation nachgeordneter Alveolen führt. Die Folge ist ein Absinken des VentilationsPerfusionsquotienten in diesen Lungenabschnitten mit einer Verschlechterung der Oxygenierung. Dadurch erklärt sich die im Durchschnitt schlechtere Sauerstoffsättigung bei adipösen Patienten im Vergleich zu einem normalgewichtigen Kollektiv. Kommt es durch Rückenlage und einen narkosebedingten Verlust des Muskeltonus zu einer weiteren Abnahme der FRC, kann der dauerhafte Verschluss kleiner Atemwege die Folge sein, was unweigerlich zur Entstehung von Atelektasen führt. Daher sollte während der Präoxygenierung und Beatmung die FRC durch eine Anhebung des exspiratorischen Atemwegsdruckes (continuous positive airway pressure [CPAP] oder positiv endexspiratorischer Atemwegsdruck [PEEP]) vergrößert werden. Sind Atelektasen be- reits entstanden, können diese nur durch einen hohen transpulmonalen Druck wieder eröffnet werden. Dazu ist entweder eine vertiefte Einatmung nötig (intensive Physiotherapie!) oder bei beatmeten Patienten die Durchführung eines Rekrutierungsmanövers. Dieses kann auch bei adipösen Patienten in herkömmlicher Weise mit einem Atemwegsdruck von $40 \mathrm{CmH}_{2} 0$, welcher für 7-8 Sekunden aufrechterhalten wird, durchgeführt werden. Um Derekrutierung vor der Extubation zu vermeiden, sollte niemals während des Extubationsvorganges endotracheal abgesaugt werden. Bewährt hat sich die Extubation nach einer vertieften Einatmung, die mit einem CPAP-Druck von etwa $30 \mathrm{CmH}_{2} \mathrm{O}$ unterstützt werden sollte.

\subsection{Veränderungen des respiratorischen Systems bei Adipositas}

\subsubsection{Lungenfunktionsparameter}

Adipositas führt zu einer gewichtsabhängigen $\mathrm{Ab}$ nahme der funktionellen Residualkapazität (Pelosi et al. 1998, s. Abb. 1) und des exspiratorischen Reservevolumens.

Diese bereits seit längerem bekannten Auswirkungen von Übergewicht auf die Lungenfunktion wurden auch in einer aktuellen Untersuchung an 149 adipösen Patienten ohne schwerwiegende Komorbiditäten (BMI $45 \pm 6$ ) bestätigt (Gabrielsen et al. 2011). Dabei lagen die dynamischen spirometrischen Parameter (forcierte Vitalkapazität und forcierte exspiratori- 


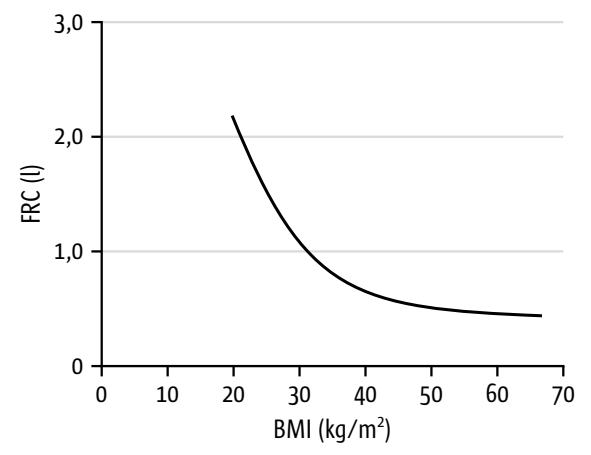

Abb. 1 Die FRC nimmt mit zunehmendem Übergewicht exponentiell ab. (nach Pelosi et al. 1998)

sche Einsekundenkapazität) sowie die Diffusionskapazität, das Residualvolumen, die Vitalkapazität und die Totalkapazität im Normbereich, während die FRC auf etwa $78 \%$ und das ERV auf etwa $50 \%$ des Normwertes vermindert waren.

Die individuellen Blutgaswerte schwankten erheblich mit $\mathrm{PaO}_{2}$-Werten zwischen 58 und $115 \mathrm{mmHg}$ und $\mathrm{PaCO}_{2}$-Werten zwischen 27 und $49 \mathrm{mmHg}$. Dabei bestanden durchweg hochsignifikante $(\mathrm{p}<\mathrm{o}, \mathrm{ol})$ wenn gleich nur schwache Korrelationen (Korrelationskoeffizienten zwischen 0,2 und $o, 4$ ) zwischen der $\mathrm{Ab}$ nahme des $\mathrm{PaO}_{2}$ bzw. der Zunahme des $\mathrm{PaCO}_{2}$ und dem BMI, dem Hüftumfang und dem Halsumfang. Diese Daten stimmen insgesamt gut mit älteren bereits publizierten Werten überein, allerdings können bei Patienten mit Obesitas Hypoventilationssyndrom der Abfall von FRC und ERV erheblich stärker ausfallen als oben angegeben.

\section{Adipositas führt zu einer gewichtsabhängigen Abnah- me von FRC und exspiratorischem Reservevolumen.}

Neben dem Ausmaß des Übergewichts ist der Fettgewebsverteilung im Hinblick auf respiratorische Störungen in der Vergangenheit eine große Bedeutung zugeschrieben worden.

So wird eine gynäkoide von einer androiden Fettverteilung unterschieden. Gynäkoid bedeutet in diesem Zusammenhang, dass sich Fettgewebe verstärkt im Bereich der Oberschenkel, des Gesäßes und der Oberarme befindet, wohingegen von einer androiden Fettverteilung gesprochen wird, wenn Fettgewebe vermehrt am Körperstamm (Brustwand, Bauchdecke und intraperitoneal) nachweisbar ist.
Adipositas vom androiden Verteilungstyp soll im Gegensatz zum gynäkoiden Typ nicht nur mit einem deutlich stärker erhöhten Risikio für Herz-Kreislauferkrankungen einhergehen, sondern auch die Lungenfunktion stärker beeinträchtigen. Die einfach nachvollziehbare, mechanistische Vorstellung geht in diesem Zusammenhang davon aus, dass die Fettmassen des Brustkorbs, der Bauchwand sowie das intraperitoneale Fett zu einer Kompression des Thorax und dadurch zu einer Verschiebung der Atemruhelage in Richtung Exspiration führen, was als Abnahme der FRC und des exspiratorischen Reservevolumens bei erhaltener Vitalkapazität nachweisbar ist (Salome et al. 2010). Die Bedeutung der Fettverteilung im Hinblick auf respiratorische Störungen ist allerdings nicht ganz unumstritten: In einer Untersuchung mittels Ganzkörper-MRT von normal- und übergewichtigen Männern und Frauen war die prozentuale Fettverteilung (Körperstamm versus Peripherie sowie Differenzierung des Körperstammfetts in thorakales und abdominales Fett, sowie anterior und posterior gelegenes subkutanes und intraabdominelles Fett) überraschender Weise bei den normal- und übergewichtigen Probanden als auch den Männern und Frauen ähnlich (Babb et al. 2008).

Die Waist/Hip Ratio (WHR), also das Verhältnis zwischen Körperumfang im Bereich der Hüfte und Taille wird of tmals benutzt, um eine androide von einer gynäkoiden Fettverteilung abzugrenzen, wobei eine WHR $\leq 0,95$ eine gynäkoide und eine WHR $>0,95$ eine androide Fettverteilung charakterisiert. In der Untersuchung von Babb und Mitarbeitern (2008) betrug die WHR allerdings trotz einer insgesamt ähnlichen Fettgewebsverteilung bei adipösen Frauen 0,85 und bei adipösen Männern o,97. Auch korrelierte in dieser Untersuchung die Abnahme der FRC mit der WHR nicht besser als mit dem BMI, dem thorakalen, abdominalen, intra- oder extraperitonealen Fettgewebeanteil.

\subsubsection{Atemmechanik}

Bei Adipositas ist die Compliance (Dehnbarkeit) des respiratorischen Systems (Crs) herabgesetzt, und gleichzeitig ist der Atemwegswiderstand erhöht (Naimark u. Cherniack 1960; Pelosi et al. 1996). Daraus resultiert zwangsläufig, dass adipöse Patienten eine höhere Atemarbeit leisten müssen als normalgewichtige $\mathrm{Pa}-$ tienten (Naimark u. Cherniack 196o; Pelosi et al. 1996). 
Adipöse Patienten müssen durch die Abnahme der Compliance und Zunahme der Resistance eine erhöhte Atemarbeit leisten.

Eine nahe liegende Erklärung für die verminderte Compliance ist der Druck, den die „Fettmassen“ auf den Brustkorb ausüben und somit dessen Ausdehnung behindern. Überraschender Weise gibt es aber widersprüchliche Befunde im Hinblick auf das Ausmaß der Verminderung der Thoraxwand compliance bei Adipositas (Pelosi et al. 1998; Naimark u. Cherniack 1960; Pelosi et al. 1996). Dagegen ist die Compliance des Lungengewebes (CL) in praktisch allen Untersuchungen erheblich reduziert. Erklärt wird die verminderte CL durch die Reduktion der FRC (s.o.) und eine damit einhergehende Zunahme der alveolären Oberflächenspannung, ein erhöhtes intrapulmonales Blutvolumen und den Verschluss von kleinen Atemwegen(s.u.) mitder Ausbildung von Atelektasen (Salome et al. 2010).

Der Atemwegswiderstand (Rrs) ist bei Adipositas vermutlich in erster Linie eine Folge der reduzierten FRC, da das Kaliber der Atemwege proportional zum Lungenvolumen zu- und abnimmt. Dementsprechend wird der absolute Rrs-Wert in den meisten Untersuchungen bei adipösen Patienten als vermindert angegeben, in Relation zum (verminderten) Lungenvolumen ist er jedoch durchaus noch normal. Durch den Verschluss von kleinen Atemwegen in der Exspiration (s.u.) wird eine zusätzliche Widerstandserhöhung zu Beginn der Inspiration hervorgerufen.

Allerdings scheint die Adipositas auch ein unabhängiger Risikofaktor für akut und chronisch obstruktive Atemwegserkrankungen zu sein (Sood 2010), da Fettgewebe über die Regulation von Leptinen und Adiponektinen die Konzentrationen von pro- und antiinflammatorischen Zytokinen beeinflusst.

\subsubsection{Airway closure- Verschluss der kleinen Atemwege}

Die Bronchiolen werden durch die radiär wirkenden Zugkräfte des Lungenparenchyms offen gehalten. Mit zunehmendem Lungenvolumen steigen diese Zugkräfte an und führen somit zu einer Erweiterung der Bronchiolen. Umgekehrt nimmt das Kaliber der kleinen Atemwege in der Exspiration ab, wodurch verständlich wird, dass sich Erkrankungen der kleinen Atemwege primär als Behinderung der Exspiration manifestieren (z.B. exspiratorische Flowlimitierung mit Giemen bei COPD). Unterschreitet das
Lungenvolumen einen kritischen Wert, tritt ein Kollaps (Verschluss) der kleinen Atemwege auf. Das Lungenvolumen, ab dem ein Verschluss der Bronchiolen nachweisbar ist, wird als Closing Capacity bezeichnet, welches mit zunehmendem Lebensalter ansteigt (ca. o,5\% der individuellen Vitalkapazität/ Jahr) (Leblanc et al. 1970). Das Lungenvolumen, welches nach Erreichen der Closing capacity noch aktiv ausgeatmet werden kann, ist das Closing volume.

Closing capacity: Lungenvolumen ab dem ein Verschluss der kleinen Atemwege nachweisbar ist. Die Closing Capacity steigt mit zunehmendem Lebensalter an.

Closing volume: Lungenvolumen das nach Erreichen der Closing capacity noch ausgeatmet werden kann.

Die FRC eines Erwachsenen ist weitgehend unabhängig vom Lebensalter (Wahba 1991; Ibanez u. Raurich 1982) und nimmt fast linear mit der Körpergröße eines Menschen zu. Übergewicht führt - wie bereits oben beschrieben - zu einer gewichtsabhängigen Reduktion der FRC. Bei gesunden normalgewichtigen Menschen ist bis zu einem mittleren Alter (50-60 J) die FRC größer als die Closing Capacity. Ein Verschluss der kleinen Atemwege tritt daher im Stehen oder Sitzen nicht auf.

Die funktionellen Residualkapazität FRC bezeichnet das Lungenvolumen am Ende einer normalen Ausatmung, wenn die entgegengesetzt wirkenden elastischen Rückstellkräfte von Lunge und Brustkorb sich gegenseitig aufheben. Im Sitzen oder Stehen beträgt die FRC eines gesunden, normalgewichtigen Erwachsenen ca. 30-35 ml/kg und bleibt unabhängig vom Alter weitgehend konstant (s. Abb. 2).

Dagegen kann bei stark übergewichtigen Menschen die deutlich reduzierte FRC (s.o.) bereits während der normalen Ruheatmung die Closing Capacity unterschreiten (Farebrother et al. 1974), sodass ein Verschluss kleiner Atemwege nachweisbar wird. Dadurch sinkt das Ventilations-Perfusionsverhältnis $\left(\dot{\mathrm{V}}_{\mathrm{A}} / \dot{\mathrm{Q}}\right)$ in den Alveolen distal des Atemwegsverschlusses, und die Oxygenierung des pulmonalkapillären Blutes im Bereich dieser Alveolen verschlechtert sich. Der erniedrigte $\mathrm{PaO}_{2}$ bei adipösen Patienten korreliert dementsprechend mit dem Ausmaß des Airway closure (Farebrother et al. 1974).

Bei adipösen Patienten kann während normaler Ruheatmung die Closing Capacity unterschritten werden, sodass es zum Verschluss von kleinen Atemwe- 


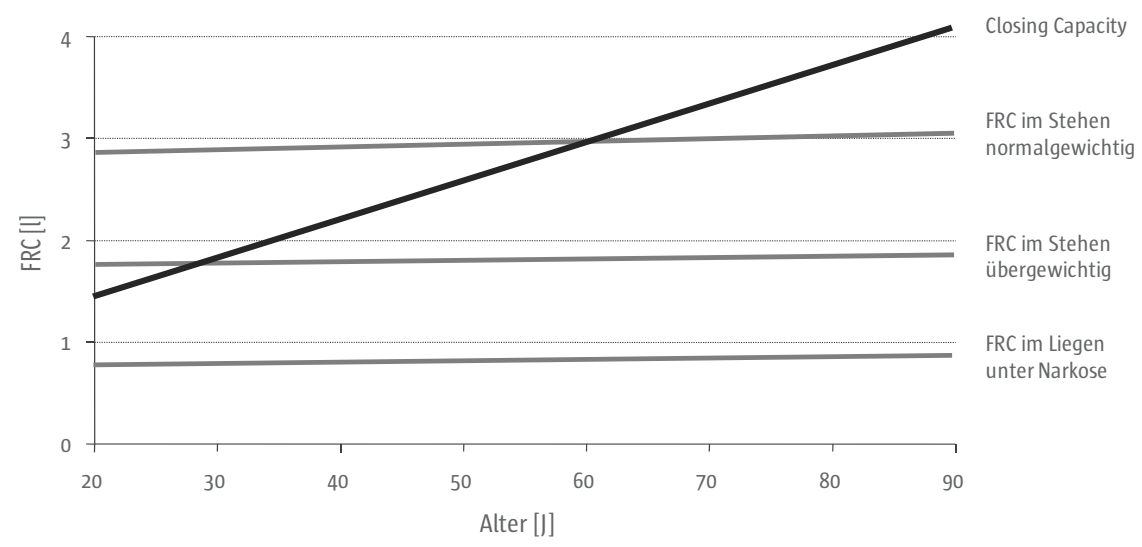

Abb. 2 Verhältnis von FRC zur Closing Capacity mit zunehmendem Lebensalter bei Normal- und Übergewicht. Die funktionelle Residualkapazität nimmt beim Erwachsenen in Abhängigkeit vom Lebensalter allenfalls geringfügig zu, während die Closing Capacity pro Lebensjahr um ca. 0,5\% der individuellen Vitalkapazität ansteigt (Leblanc et al. 1970). Dadurch kann es unter Ruheatmung auch bei Normalgewichtigen bereits um das 50. Lebensjahr zum Verschluss der kleinen Atemwege während der Exspiration kommen. Bei Adipositas ist die FRC gewichtsabhängig bereits im Stehen und Sitzen erheblich vermindert. Durch die zusätzliche Abnahme der FRC in Rückenlage und nochmalige Reduktion der FRC in Allgemeinanästhesie, überschreitet die Closing Capacity die FRC sogar schon bei jungen Erwachsenen deutlich. Daraus kann ein dauerhafter Verschluss der kleinen Atemwege unter Narkose resultieren.

gen kommt (Airway closure). Der daraus resultierende Abfall des Ventilations-Perfusionsverhältnisses nachgeordneter Alveolen erklärt u.a. die Oxygenierungsstörung bei Adipositas.

Besondere Bedeutung bekommt das Phänomen des Airway closure im Zusammenhang mit der Einleitung einer Allgemeinanästhesie, da die FRC in Rückenlage im Vergleich zum Sitzen oder Stehen um etwa 25\% reduziert ist (Lumb u. Nunn 1991) und durch die Muskelrelaxation um weitere $15-20 \%$ abnimmt (Wahba 1991). Die Folge eines Atemwegsverschluss ist zunächst wie oben beschrieben eine verminderte Exspiration von Atemgas aus den nachgeschalteten Alveolen (,Airtrapping“). Dieses Airtrapping behindert die nachfolgende Inspiration und führt dadurch zu einer Abnahme des regionalen Tidalvolumens und des $\dot{\mathrm{V}}_{\mathrm{A}} / \dot{\mathrm{Q}}$-Quotienten in diesen Lungenarealen. Der $\dot{\mathrm{V}}_{\mathrm{A}} / \dot{\mathrm{Q}}$-Quotient wiederum ist die entscheidende Determinante für die Oxygenierungsleistung einer funktionellen Lungeneinheit, wobei ein Abfall des $\dot{\mathrm{V}}_{\mathrm{A}} / \dot{\mathrm{Q}}$-Quotienten mit einem Abfall des $\mathrm{PaO}_{2}$ einhergeht.

Die FRC vermindert sich in Rückenlage um ca. $25 \%$ und wird nach Narkoseeinleitung um weitere $20 \%$ reduziert.
Bleiben die kleinen Atemwege nicht nur am Ende der Exspiration verschlossen sondern während des gesamten Atemzyklus, können sich bereits nach kurzer Zeit (wenige Minuten) Resorptionsatelektasen bilden. Die Geschwindigkeit der Entstehung von Resorptionsatelektasen hängt u.a. von der Zusammensetzung des Atemgases ab (Rothen et al. 1995). Je besser die Löslichkeit eines Gases im Blut und je höher die Partialdruckdifferenz zwischen Blut und Alveolarluft ist, desto schneller wird das Gas aus den Alveolen resorbiert. Diese beiden Aspekte wirken sich im Rahmen der Narkoseeinleitung während der Präoxygenierungsphase besonders ungünstig aus.

\section{Aus Sicherheitsgründen sollte zur Narkoseeinleitung reiner Sauerstoff über eine dicht sitzende Maske ver- abreicht werden, damit während der Apnoephase vor Intubation eine möglichst große Menge Sauerstoff in der Lunge gespeichert ist.}

Im Falle einer ,cannot ventilate cannot intubate“ Situation errechnet sich die tolerable Apnoezeit als (FRC $\mathrm{x}$ alveoläre $\mathrm{O}_{2}$-Konzentration/10o)/,, $\mathrm{O}_{2}$-Verbrauch in Ruhe“. Veranschlagt man für den $\mathrm{O}_{2}$-Verbrauch näherungsweise $3 \mathrm{ml} / \mathrm{kg} / \mathrm{min}$ und beträgt die FRC bei einem Normalgewichtigen etwa $30 \mathrm{ml} / \mathrm{kg}$, so ergibt sich bei kompletter Füllung der FRC mit Sauerstoff eine 
Apnoetoleranz von etwa 10 min. Bei adipösen Patienten kann die FRC in Narkose je nach BMI auf Werte von nur noch 3-5 ml/kg reduziert sein (Pelosi et al. 1999), sodass die Apnoetoleranz selbst bei perfekter Präoxygenierung auf 1-2 Minuten reduziert ist. Daher ist eine ausgiebige Präoxygenierung mit $100 \% \mathrm{O}_{2}$ und dicht sitzender Maske während der Narkoseeinleitung bei adipösen Patienten zwingend erforderlich!

Bei perfekter Präoxygenierung beträgt die Apnoetoleranz eines Normalgewichtigen etwa 10 Minuten, während sie bei Adipösen je nach BMI auf 1-2 Minuten reduziert sein kann. Daher ist eine ausgiebige Präoxygenierung mit $100 \% \mathrm{O}_{2}$ und dicht sitzender Maske während der Narkoseeinleitung bei adipösen Patienten zwingend erforderlich, obwohl dadurch die Entstehung von Resorptionsatelektasen gefördert wird.

Sauerstoff ist im Blut etwa doppelt so gut löslich wie Stickstoff (Nunn 1993) und die Partialdruckdifferenz für $\mathrm{O}_{2}$ zwischen Alveolen und pulmonalkapillärem Blut beträgt nach „perfekter Präoxygenierung“ etwa 6oo mmHg! Dadurch kommt es nach Einatmung von reinem Sauerstoff und dem Verschluss kleiner Atemwege zu einer sehr schnellen Ausbildung von Resorptionsatelektasen. Modellrechnungen zur Resorptionsgeschwindigkeit von Gasen aus einem verschlossenen Lungenkompartiment zeigen einen exponentiellen Abfall der Resorptionsdauer mit zunehmender inspiratorischer $\mathrm{O}_{2}$-Konzentration (Joyce $\mathrm{u}$. Williams 1999). Auch in klinischen Untersuchungen bestätigte sich, dass die Entstehung von Atelektasen sowohl während der Narkoseeinleitung (Edmark et al. 2003) als auch während einer Beatmung (Rothen et al. 1995) von der inspiratorischen Sauerstoffkonzentration abhängt. Interessanter Weise führt aber bereits eine Reduktion der inspiratorischen Sauerstoffkonzentration von $100 \%$ auf $80 \%$ während der Präoxygenierung zu einer annähernd vollständigen Vermeidung der Atelektasenbildung (Edmark et al. 2003).

Der Stickstoffanteil $\left(\mathrm{N}_{2}\right)$ in der Atemluft wird nur langsam aus den Alveolen resorbiert, da $\mathrm{N}_{2}$ im Blut schlechter löslich ist als $\mathrm{O}_{2}$ und bereits nach einer kurzen Präoxygenierungsphase der $\mathrm{N}_{2}$-Partialdruck im gemischtvenösen Blut höher ist als der alveoläre Stickstoffpartialdruck, sodass kein Diffusionsgradient von der Alveolarluft ins Blut besteht. Kommt es allerdings zu einem dauerhaften Verschluss von Bronchiolen, bilden sich unabhängig von der einge- atmeten Gasmischung im Laufe der Zeit unweigerlich Atelektasen (Joyce u. Williams 1999).

Die oben dargelegten Zusammenhänge legen die theoretische Schlussfolgerung nahe, bei adipösen Patienten möglichst niedrige inspiratorische Sauerstoffkonzentrationen zu verwenden. Da Lachgas etwa um den Faktor 16 besser im Blut löslich ist als Sauerstoff, muss die intraoperative Verwendung eines Sauerstoff-Lachgas-Gemisches zur Beatmung bei adipösen Patienten besonders kritisch überdacht werden.

Eine Beatmung mit reinem Sauerstoff und einem Sauerstoff-Lachgasgemisch begünstigt besonders die Entstehung von Resorptionsatelektasen, allerdings ist die reduzierte Fähigkeit der Lunge zur Sauerstoffspeicherung bei Adipösen zu bedenken (s.o.)

\subsubsection{Atelektasen}

Atelektasen sind kollabierte oder mit Flüssigkeit gefüllte Alveolen. Bei lungengesunden, wachen, spontan atmenden Patienten sind Atelektasen praktisch nicht nachweisbar und adipöse Patienten haben im Wachzustand allenfalls minimale Atelektasen (Coussa et al. 2004; Reinius et al. 2009). Dagegen finden sich unmittelbar nach Narkoseeinleitung Atelektasen bei ca. 90\% aller Patienten in den dorsalen Lungenabschnitten (Gunnarson et al. 1991) (s. Abb. 3).

Adipöse Patienten sind durch ihre verminderte FRC
und das sich daraus ergebende Phänomen des Air-
way closure besonders gefährdet, während der Nar-
koseeinleitung und einer nachfolgenden maschinel-
len Beatmung Atelektasen zu entwickeln (Rothen et
al. 1993; Coussa et al. 2004); die Atelektasen sind
größer als bei normalgewichtigen Patienten.

Während bei lungengesunden Patienten, die sich einer Allgemeinanästhesie mit kontrollierter Beatmung unterziehen, in computertomografischen Schnittbildern der basalen Lungenabschnitte meistens nur zwischen $1 \%$ und $5 \%$ der Lungenfläche atelektatisch sind (Lundquist et al. 1995), kann dieser Anteil auf $10-15 \%$ des Lungengewebes bei übergewichtigen Patienten erhöht sein (Rothen et al. 1993; Coussa et al. 2004; Reinius et al. 2009). Dabei muss zusätzlich bedacht werden, dass kollabierende Alveolen auf etwa 20\% ihres Ausgangsvolumens zusammenschrumpfen. 


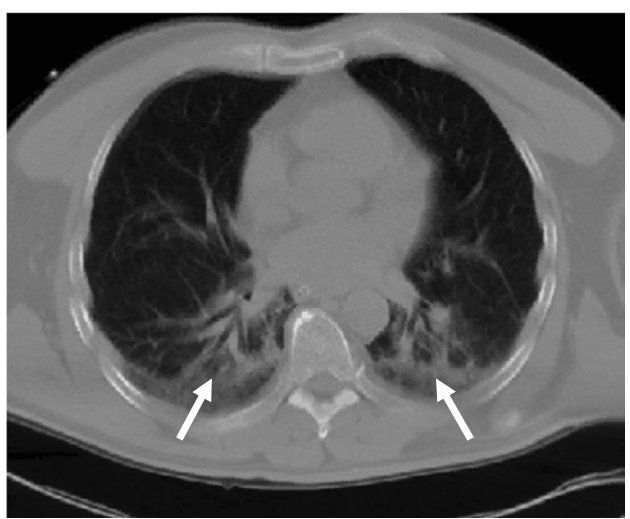

Abb. 3 CT nach Narkoseeinleitung und Intubation. Bereits wenige Minuten nach Narkoseeinleitung finden sich bei ca. 90\% aller Patienten dorsobasale Atelektasen (Pfeile). Ursächlich hierfür sind u.a. die Präoxygenierung und Beatmung mit reinem Sauerstoff sowie die Reduktion der FRC durch Rückenlagerung, tiefe Sedierung und Muskelrelaxierung. Bei adipösen Patienten sind diese Atelektasen besonders ausgeprägt.

So können nach Narkoseeinleitung tatsächlich bis zu $50 \%$ des Lungenparenchyms in den basalen $\mathrm{Ab}$ schnitten atelektatisch sein.

Das Ausmaß der Atelektasen korreliert dabei invers mit der Höhe des $\mathrm{PaO}_{2}$ unter Beatmung (Neumann et al. 1999, s. Abb. 4). Atelektasen führen zu einem intrapulmonalen Rechts-Links-Shunt, da das gemischtvenöse Blut der Lungenarterien in den Atelektasen nur luftleere, nicht ventilierte Alveolen umspült, und mit der Atemluft nicht in Kontakt kommt. Dieses Blut nimmt am Gasaustausch in der Lunge nicht teil (Shunt) und führt zu einer Reduktion des Sauerstoffgehaltes im pulmonal-venösen und dann arteriellen Blut.

Der Versuch, die 0xygenierung eines Patienten mit einem intrapulmonalen Shunt durch die Gabe von $\mathrm{O}_{2}$ nachhaltig zu verbessern, muss also scheitern!

Die klinische Erfahrung, dass sich dennoch bei den meisten Patienten die Oxygenierung durch $\mathrm{O}_{2}$-Gabe steigern lässt, liegt in der Tatsache begründet, dass neben Atelektasen mit Shunt immer auch schlecht ventilierte Alveolarbezirke (Low- $\dot{V}_{A} / \dot{Q}^{-}$Bezirke, s.u.)

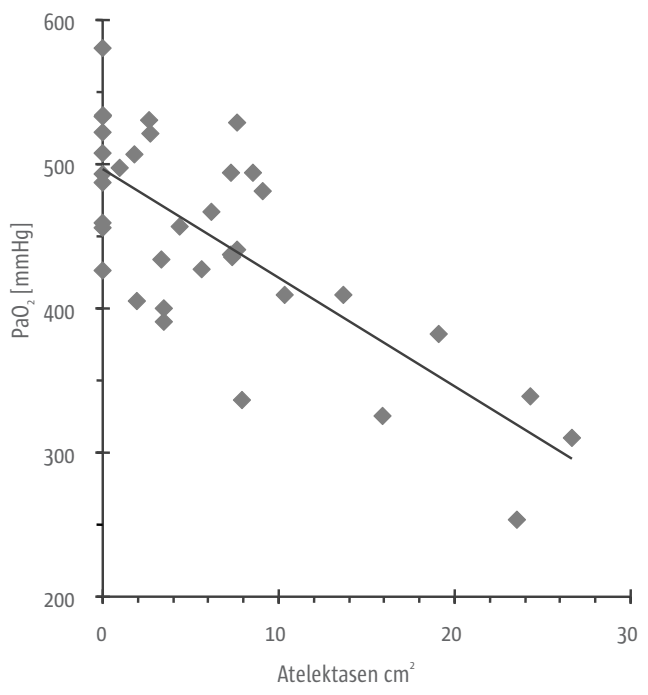

Abb. 4 Rekrutierungsmanöver. Die X-Achse zeigt die Größe der Atelektasen eines transversalen CT-Schnittbildes in $\mathrm{cm}^{2}$. Auf der Y-Achse sind die $\mathrm{PaO}_{2}$-Werte in $\mathrm{mmHg}$ aufgetragen. $\mathrm{r}^{2}=0,57$ (nach Neumann et al. 1999)

zu einer Oxygenierungsstörung beitragen. Das Verhältnis von Shunt $z u$ Low- $\dot{V}_{\mathrm{A}} / \dot{Q}$ ist letztlich entscheidend, wie sehr ein Patient mit einer Oxygenierungsstörung von einer alleinigen $\mathrm{O}_{2}$-Gabe profitiert. Bei Normalgewichtigen steigt der intrapulmonale Shunt von < $1 \%$ des Herzzeitvolumens $\left(\dot{\mathrm{Q}}_{\mathrm{T}}\right)$ unter Spontanatmung im Wachzustand auf ca. $5 \%$ während einer Allgemeinanästhesie an. Bei adipösen Patienten entspricht das Ausmaß der Oxygenierungsstörung nach Narkoseeinleitung einem Shuntanteil von biszu $25 \%$.

Die Wahl des Narkotikums ist wahrscheinlich ohne wesentliche Bedeutung für das Ausmaß der Atelektasenbildung.

Nur bei einer Spontanatmungsnarkose mit Ketamin treten bei normalgewichtigen Menschen Atelektasen praktisch nicht auf, wohingegen bei der Kombination von Ketamin mit einem Muskelrelaxans das Ausmaß der Atelektasenbildung identisch im Vergleich zur Anwendung anderer Narkotika ist (Tokics et al. 1987). Für adipöse Patienten liegen bislang keine Untersuchungen vor, in denen der Einfluss unterschiedlicher Narkotika auf die Entstehung von Atelektasen untersucht wurde. 


\subsection{Therapiemaßnahmen zur Verbesserung des Gasaustausches}

Die entscheidenden Therapiemaßnahmen zur Verbesserung des gestörten Gasaustausches bei Adipositas haben die Vergrößerung der pathologischen verminderten FRC und damit die Vermeidung von Atelektasen bzw. die Rekrutierung von Lungenparenchym zum Ziel. Während einer Beatmung entstehen Atelektasen primär während der Exspirationsphase, wenn das Lungenvolumen die Closing Capacity unterschreitet und der transpulmonale Druck (Differenz zwischen dem intraalveolären Druck und dem Pleuradruck), welcher für die Ausdehnung der Lunge entscheidend ist, niedrig ist. Eine Anhebung des endexspiratorischen Lungenvolumens ist durch die Verwendung von PEEP möglich.

Eine Wiedereröffnung kollabierter Lungenareale ist dagegen ein inspiratorisches Phänomen, das immer dann eintritt, wenn der kritische Eröffnungsdruck einer Alveole überschritten wird. Dieser kritische Eröffnungsdruck ist in verschiedenen Bereichen der Lunge regional unterschiedlich hoch und variiert zusätzlich je nach der für die Atelektasenbildung zugrunde liegenden Lungenpathologie. Daher können verschlossene Alveolen einerseits bereits während einer normalen Inspiration rekrutiert werden, während andererseits Alveolen trotz der Anwendung exzessiv hoher Atemwegsdrücke verschlossen bleiben können.

\section{Verwendung von CPAP und PEEP}

Die Vergrößerung der FRC durch die Aufrechterhaltung eines kontinuierlichen positiven Atemwegsdruck (CPAP) während der Präoxygenierung in Kombination mit einer Maskenbeatmung mit PEEP führt bei adipösen Patienten zu einer signifikanten Reduktion der Atelektasenbildung während der Narkoseeinleitung (Coussa et al. 2004). Eine teilweise Stabilisierung der FRC und Verbesserung der Oxygenierung im Vergleich zu einer Kontrollgruppe konnte bei adipösen Patienten während der Narkoseeinleitung auch durch eine maschinelle druckunterstützte Spontanatmung mit PEEP erreicht werden (Futier et al. 2011). Dabei stellt sich zwangsläufig die Frage, welches PEEP-Niveau erforderlich ist, um eine Atelektasenbildung zu vermeiden. Talab und Mitarbeiter (2009) veröffentlichten 2009 eine Arbeit, in der sie nach einem Rekrutierungsmanöver (s.u.) ein PEEP von $\mathrm{o}, 5$ und $10 \mathrm{CmH}_{2} \mathrm{O}$ bei Patienten mit einem BMI zwischen 30 und 50 anwandten. Selbst durch ein PEEP-Niveau von $10 \mathrm{CmH}_{2} \mathrm{O}$ ließen sich eine Atelektasenbildung und erhöhte alveolo-arterielle Sauerstoffdifferenz nicht vollständig vermeiden, aber diese Phänomene waren weniger stark ausgebildet als in den PEEP $=5 \mathrm{cmH}_{2} \mathrm{O}$ und PEEP $=\mathrm{O} \mathrm{cmH}_{2} \mathrm{O}$ Gruppen.

\section{Während einer Narkoseeinleitung kann die Entstehung von Atelektasen durch CPAP und/oder PEEP reduziert werden. Dabei sollte ein PEEP-Niveau $\geq 10 \mathrm{Cm} \mathrm{H}_{2} \mathrm{O}$ ver- wendet werden.}

Zur Wiedereröffnung kollabierter Alveolen ist ein hoher transpulmonaler Druck (Eröffnungsdruck) erforderlich, der während einer normalen Inspiration nicht zwangsläufig erreicht wird. Daher führt die alleinige Beatmung mit PEEP nicht automatisch zur Rekrutierung von Lungenparenchym und Verbesserung des Gasaustausches (Reinius et al. 2009; Futier et al. 2011). Bei gesunden Erwachsenen muss perioperativ ein Atemwegsdruck von $40 \mathrm{CmH}_{2} \mathrm{O}$ angewendet werden, um eine annähernd vollständige Rekrutierung von Atelektasen zu erreichen (Rothen et al. 1993). Dieser Druck sollte für eine Dauer von 7 Sekunden (Rothen et al. 1999) gehalten werden (s. Abb. 5). Auch bei stark übergewichtigen Patienten ist ein Rekrutierungsmanöver mit einem Atemwegsdruck von $40 \mathrm{cmH}_{2} \mathrm{O}$, der über 7-8 Sekunden aufrecht gehalten wird, effektiv (Talab et al. 2009), wenngleich auch längere Rekrutierungsmanöver (40 s) und die Verwendung höhere Atemwegsdrücke bis $55 \mathrm{cmH}_{2} \mathrm{O}$

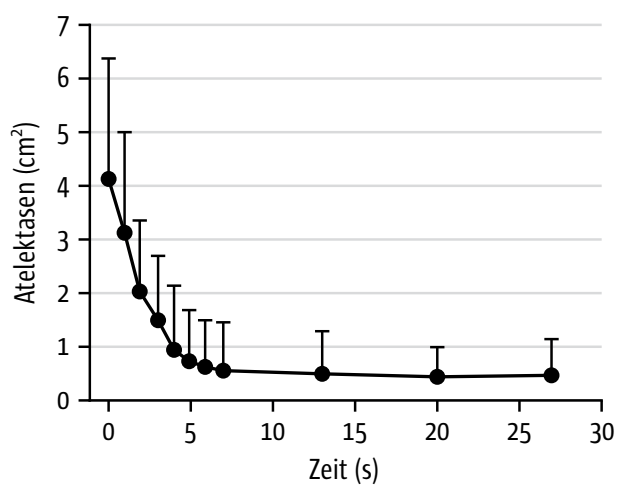

Abb. 5 Rekrutierungsmanöver. Die Aufrechterhaltung eines Atemwegsdruckes von ca. $40 \mathrm{~cm} \mathrm{H}_{2} \mathrm{O}$ führt bereits nach 7 Sekunden zu einer fast vollständigen Rekrutierung von perioperativ entstandenen Atelektasen. (nach Rothen et al. 1999) 
(Reinius et al. 2009) beschrieben wurden. Da die Reduktion von Atelektasen einer exponentiellen Kinetik folgt (Rothen et al. 1999), ist von länger andauernden Rekrutierungsmanövern keine nennenswerte zusätzliche Wiedereröffnung von Atelektasen zu erwarten. Durch den hohen intrathorakalen Druck tritt während des Rekrutierungsmanövers aber bei den meisten Patienten eine Blutdrucksenkung auf. Daher sollte jedes Rekrutierungsmanöver grundsätzlich nur so kurz wie nötig sein. Gefährlich können die oben beschriebenen Maßnahmen insbesondere bei hypovolämen oder kardial erkrankten Patienten sein, die nicht selten Arrhythmien und Bradykardien während oder kurz nach dem Rekrutierungsmanöver entwickeln. Eine inflammatorische Reaktion mit sekundärer Lungenschädigung ist dagegen als Folge der oben beschriebenen Manöver nicht zu erwarten (Puls et al. 2006).

Für normal- und übergewichtige lungengesunde $\mathrm{Pa}$ tienten ist ein perioperativ durchgeführtes Rekrutierungsmanöver mit einem Atemwegsdruck von $40 \mathrm{cmH}_{2} 0$, welcher für 7 Sekunden aufrechterhalten wird, ausreichend.

Ein solches Rekrutierungsmanöver darf aber nur bei normovolämen Patienten ohne schwerwiegende kardiale Vorerkrankungen durchgeführt werden, da anderenfalls nicht selten bedrohliche Blutdruckabfälle, ventrikuläre Arrhythmien und Bradykardien auftreten!

Da ein Rekrutierungsmanöver nur zur Wiedereröffnung verschlossener Alveolen führt, jedoch nicht das erneute Auftreten von Atelektasen verhindert (Neumann et al. 1999; Reinius et al. 2009), sollte nach einer erfolgreichen Rekrutierung immer PEEP zur Beatmung verwendet werden.

\subsection{Narkoseführung bei adipösen Patienten}

Während der Narkoseeinleitung sind adipöse Patienten in besonderer Weise gefährdet eine Hypoxämie zu erleiden. Ursächlich ist zum einen die geringere FRC und damit auch eine geringere Sauerstoffreserve im Vergleich zu normalgewichtigen Patienten. Die Apnoetoleranz (Zeitintervall bis zum Abfall der Sauerstoffsättigung $<90 \%$ ) wird daher mit zunehmendem Übergewicht kürzer. Gleichzeitig weisen adipöse Patienten vergleichsweise häufig erschwerte Intubationsbedingungen auf. Daher ist eine ausgiebige Präoxygenierung mit dicht sitzender Maske unabdingbar. Zur
Vergrößerung der FRC sollte während der Präoxygenierung ein CPAP-Druck von etwa $10 \mathrm{cmH}_{2} \mathrm{O}$ aufrechterhalten und nach Eintreten der Apnoe die Beatmung mit PEEP $\sim 10 \mathrm{cmH}_{2} \mathrm{O}$ bis zur Intubation fortgesetzt werden. Durch diese Maßnahmen lässt sich ein Abfall der Sauerstoffsättigung fast immer vermeiden.

Auch nach der endotrachealen Intubation sollte die Entstehung von Atelektasen durch Beatmung mit PEEP von ca. $10 \mathrm{CmH}_{2} \mathrm{O}$ vermieden werden. Kommt es dennoch zu einer kontinuierlichen Verschlechterung des Gasaustausches während der Beatmung oder ist der Gasaustausch bereits unmittelbar nach Narkoseeinleitung erheblich gestört, müssen zuerst typische Ursachen wie eine einseitige Intubation oder ein Pneumothorax nach zentraler Venenkatheteranlage ausgeschlossen werden. Danach kann ein Rekrutierungsmanöver durchgeführt werden, indem ein Atemwegsdruck von $40 \mathrm{CmH}_{2} \mathrm{O}$ für $7-8$ Sekunden aufrecht gehalten wird. Während und kurz nach dem Rekrutierungsmanöver sieht man zuerst praktisch immer einen Abfall der Sauerstoffsättigung, welcher durch eine erhöhte Sauerstoffausschöpfung bei vermindertem Herzzeitvolumen während des Rekrutierungsmanövers erklärbar ist. Erst 20-30 Sekunden später tritt eine sprunghafte Verbesserung der Sauerstoffsättigung auf. Spätestens nach dem Rekrutierungsmanöver sollte zur Beatmung ein PEEP $\geq 10 \mathrm{cmH}_{2} \mathrm{O}$ eingestellt werden.

Kommt es unter der Beatmung zu einem erneuten Derecruitment, kann das Rekrutierungsmanöver selbstverständlich wiederholt werden.

Während der Ausleitungsphase sollte, wenn immer möglich, auf die Beatmung mit reinem Sauerstoff verzichtet werden, da bereits ein Stickstoffanteil von nur $20 \%$ in der Atemluft bestehende Resorptionsatelektasen deutlich vermindert (Edmark et al. 2003). Ein solches Vorgehen erscheint auch unter Sicherheitsaspekten vertretbar, da ja bereits von der Narkoseeinleitung bekannt ist, ob die Maskenbeatmung möglich und die endotracheale Intubation erschwert ist.

Die Extubation sollte in fast sitzender Position oder zumindest mit erhöhtem Oberkörper erfolgen, da in dieser Position die FRC größer ist als in Rückenlage. Eine Unsitte ist die endotracheale Absaugung während der Extubation. In Abhängigkeit von der Größe des Endotrachealtubus und der Cröße des Absaugkatheters treten während des Absaugvorganges intrapulmonal Druckabfälle bis zu-8o mbar auf (Stenqvist et al. 2001), die zwangsläufig mit der Entstehung von Atelektasen einhergehen. Ist vor der Ausleitung sehr viel Sekret in den Atemwegen vorhanden, sollte abgesaugt und anschließend rekrutiert werden. Ist ein 
endotracheales Absaugen vor der Extubation nicht erforderlich, hat sich folgendes Vorgehen bewährt:

- Der Patient wird während der Ausleitung auf Spontanatmung mit einem CPAP von etwa $10 \mathrm{CmH}_{2} \mathrm{O}$ umgestellt.

- Der Mund- und Rachenraum wird gründlich abgesaugt.

- Unmittelbar vor der Extubation wird der Patient aufgefordert, tief einzuatmen und gleichzeitig wird der Atemwegsdruck auf $30 \mathrm{cmH}_{2} \mathrm{O}$ erhöht.

- Dann wird der Tubus entblockt, wobei durch den hohen Atemwegsdruck das Sekret, welches sich oberhalb des Cuffs angesammelt hat, mit dem Luftstrom aus der Trachea in die Mundhöhle gelangt, von wo es abgesaugt werden kann.

- Anschließend erfolgt die Extubation.

\section{Prinzipien der Narkoseführung}
- ausgiebige Präoxygenierung mit $100 \% 0_{2}$ und CPAP $10 \mathrm{CmH} O$
- bei Apnoe Maskenbeatmung mit PEEP $\sim 10 \mathrm{CmH}_{2} \mathrm{O}$
- bei Oxygenierungsstörung nach Ausschluss einer Tubusfehllage und eines Pneumothorax Rekruitmentmanöver erwägen
- Rekruitmentmanöver mit Atemwegsdruck = $40 \mathrm{CmH}_{2} 0$ für 7-8 Sekunden
- in der Ausleitungsphase FiO auf maximal 0,8 stellen
- nicht während des Extubierens absaugen
- zur Extubation tief einatmen lassen und Atemwegsdruck auf $30 \mathrm{CmH}_{2} \mathrm{O}$ erhöhen

Postoperativ sollte die Lungenfunktion frühzeitig mittels nicht-invasiver Beatmung stabilisiert und verbessert werden (Ebeo et al. 2002).

\section{Literatur}

Babb TG, Wyrick BL, Delorey DS, Chase PI, Feng MY (2008) Fat distribution and end-expiratory lung volume in lean and obese men and women. Chest 134, 704-711

Coussa M, Proietti S, Schnyder P, Frascarolo P, Suter M, Spahn DR, Magnusson L (2004) Prevention of atelectasis formation during the induction of general anesthesia in morbidly obese patients. Anesth Analg 98, 1491-1495

Edmark L, Kostova-Aherdan K, Enlund M, Hedenstierna G (2003) Optimal oxygen concentration during induction of general anesthesia. Anesthesiology 98, 28-33

Farebrother MJ, McHardy GJ, Munro JF (1974) Relation between pulmonary gas exchange and closing volume before and after substantial weight loss in obese subjects. Br Med I 3, 391-393
Futier E, Constantin JM, Pelosi P, Chanques G, Kwiatkoskwi F, Jaber S, Bazin JE (2010) Intraoperative recruitment maneuver reverses detrimental pneumoperitoneum-induced respiratory effects in healthy weight and obese patients undergoing laparoscopy. Anesthesiology 113, 1310-1319

Futier E, Constantin JM, Pelosi P, Chanques G, Massone A, Petit A, Kwiatkowski F, Bazin JE, Jaber S (2011) Noninvasive Ventilation and Alveolar Recruitment Maneuver Improve Respiratory Function during and after Intubation of Morbidly Obese Patients: A Randomized Controlled Study. Anesthesiology 114, 1354-1363

Gabrielsen AM, Lund MB, Kongerud |, Viken KE, Røislien I, Hjelmesæth I (2011) The relationship between anthropometric measures, blood gases, and lung function in morbidly obese white subjects. Obes Surg 21, 485-491

Gunnarsson L, Tokics L, Gustavsson H, Hedenstierna G (1991) Influence of age on atelectasis formation and gas exchange impairment during general anaesthesia. Br | Anaesth 66, 423-432

Ibanez |, Raurich JM (1982) Normal values of functional residual capacity in the sitting and supine positions. Intensive Care Med 8, 173-177

Joyce Cl, Williams AB (1999) Kinetics of absorption atelectasis during anesthesia: a mathematical model. J Appl Physiol 86, 11161125

Leblanc P, Ruff F, Milic-Emili ) (1970) Effects of age and body position on „airway closure“ in man. | Appl Physiol 28, 448-451

Lumb AB, Nunn JF (1991) Respiratory function and ribcage contribution to ventilation in body positions commonly used during anesthesia. Anesth Analg 73, 422-426

Lundquist H, Hedenstierna G, Strandberg A, Tokics L, Brismar B (1995) CT-assessment of dependent lung densities in man during general anaesthesia. Acta Radiol 36, 626-632

Naimark A, Cherniack RM (1960) Compliance of the respiratory system and its components in health and obesity. I Appl Physiol 15, 377-382

Neumann P, Rothen HU, Berglund JE, Valtysson I, Magnusson A, Hedenstierna G (1999) Positive end-expiratory pressure prevents atelectasis during general anaesthesia even in the presence of a high inspired oxygen concentration. Acta Anaesthesiologica Scandinavica 43, 295-301

Nunn JF (1993) Nunn's applied respiratory physiology. ButterworthHeinemann 0xford, 583-593

Pelosi P, Croci M, Ravagnan I, Vicardi P, Gattinoni L (1996) Total respiratory system, lung, and chest wall mechanics in sedatedparalyzed postoperative morbidly obese patients. Chest 109, 144-151

Pelosi P, Croci M, Ravagnan I, Tredici S, Pedoto A, Lissoni A, Gattinoni $L$ (1998) The effects of body mass on lung volumes, respiratory mechanics, and gas exchange during general anesthesia. Anesth Analg 87, 654-660

Pelosi P, Ravagnan I, Giurati G, Panigada M, Bottino N, Tredici S, Eccher G, Gattinoni L (1999) Positive end-expiratory pressure improves respiratory function in obese but not in normal subjects during anesthesia and paralysis. Anesthesiology 91, 12211231

Puls A, Pollok-Kopp B, Wrigge H, Quintel M, Neumann P (2006) Effects of a single-lung recruitment maneuver on the systemic release of inflammatory mediators. Intensive Care Med 32, 1080-1085 
Reinius H, Jonsson L, Gustafsson S, Sundbom M, Duvernoy 0, Pelosi P, Hedenstierna G, Fredén F (2009) Prevention of atelectasis in morbidly obese patients during general anesthesia and paralysis: a computerized tomography study. Anesthesiology 111, 979-987

Rothen HU, Sporre B, Engberg G, Wegenius G, Hedenstierna G (1993) Re-expansion of atelectasis during general anaesthesia: a computed tomography study. Br | Anaesth 71, 788-795

Rothen HU, Sporre B, Engberg G, Wegenius G, Högman M, Hedenstierna $G$ (1995) Influence of gas composition on recurrence of atelectasis after a reexpansion maneuver during general anesthesia. Anesthesiology 82, 832-842

Rothen HU, Neumann P, Berglund JE, Valtysson I, Magnusson A, Hedenstierna $G$ (1999) Dynamics of re-expansion of atelectasis during general anaesthesia. Br | Anaesth 82, 551-556
Salome CM, King GG, Berend N (2010) Physiology of obesity and effects on lung function. | Appl Physiol 108, 206-211

Sood A (2010) Obesity, adipokines, and lung disease. J Appl Physiol 108, 744-753

Talab HF, Zabani IA, Abdelrahman HS, Bukhari WL, Mamoun I, Ashour MA, Sadeq BB, El Sayed SI (2009) Intraoperative ventilatory strategies for prevention of pulmonary atelectasis in obese patients undergoing laparoscopic bariatric surgery. Anesth Analg 109, 1511-1516

Tokics L, Strandberg A, Brismar B, Lundquist H, Hedenstierna G (1987) Computerized tomography of the chest and gas exchange measurements during ketamine anaesthesia. Acta Anaesthesiol Scand 31, 684-692

Wahba RW (1991) Perioperative functional residual capacity. Can I Anaesth 38, 384-400

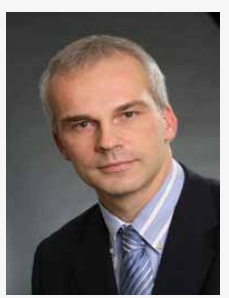

\section{Prof. Dr. Peter Neumann}

Studium der Humanmedizin an der Georg-August-Universität Göttingen, 1990 Promotion, anschließend Tätigkeit als Assistenzarzt, später Oberarzt am Zentrum für Anästhesiologie, Rettungs- und Intensivmedizin. Im Jahr 2000 Ph.D. an der Universität Uppsala im Fachbereich Klinische Physiologie, 2001 Habilitation an der Georg-August-Universität Göttingen. Von 2003 bis 2007 Leitender Oberarzt der Anästhesiologischen Abteilungen des Zentrums für Anästhesiologie, Rettungs- und Intensivmedizin. Seit 2007 Chefarzt des Instituts für Klinische Anästhesiologie des Evangelischen Krankenhauses Göttingen-Weende, Ernennung zum außerplanmäßigen Professor im gleichen Jahr. Professor Neumann ist Mitglied in diversen medizinischen Fachgesellschaften, darunter die Deutsche Gesellschaft für Anästhesiologie und Intensivmedizin (DGAI) und die Deutsche Interdisziplinäre Vereinigung für Intensivmedizin (DIVI). 